\title{
NOTE ON HADWIGER-FINSLER'S INEQUALITIES
}

\section{D.Ş. Marinescu, M. Monea, M. Opincariu and M. Stroe}

Abstract. In this article we present a new proof of the Finsler-Hadwiger inequality, we prove some consequences and one Finsler-Hadwiger type inequality. Finally we use the geometric inequalities that we obtain in this paper to present some algebraic applications.

Mathematics subject classification (2010): 26D05.

Keywords and phrases: Finsler-Hadwiger's inequality, Weitzenböck's inequality, means inequality.

\section{REFERENCES}

[1] T. Andreescu, G. Dospinescu, M. Lascu, M. Tetiva, Old and New Inequalities, GIL Publishing House, Zalau 2004.

[2] O. Bottema, R.Z. Djordjević, R.R. Janić, D.S. Mitrinović, P.M. Vasić, Geometric Inequalities, Wolters-Noordhoe Publishing, Groningen 1969.

[3] T.R. Curry, The American Mathematical Monthly, 73 (1966), no. 2, problem E 1861.

[4] P.H. Duc, An Unexpectedly Useful Inequality, Mathematical Reflections, 2008, Issue 1.

[5] A. Engel, Problem-Solving Strategies, Springer Verlag, New York, 1998.

[6] P. von Finsler, H. Hadwiger, Einige Relationen im Dreieck, Commentarii Mathematici Helvetici, 10 (1937), no. 1, 316-326.

[7] V.O. GoRdon, Matematika v Skole, 1966, No. 1, 89.

[8] H. Hadwiger, Jber. Deutsch. Math. Verein., 49 (1939), 35-39.

[9] D.S. Mitrinović, J.E. PeČARIĆ, V. VolEnEC, Recent Advances in Geometric Inequalities, Kluwer Academic Publisher, 1989.

[10] G. Tsintsifas, The American Mathematical Monthly, 93 (1986), no. 5, problem E 3150.

[11] R. Weitzenвöck, Uber eine Ungleichung in der Dreiecksgeometrie, Mathematische Zeitschrift, 5 (1919), no. 1-2, 137-146. 